\title{
Formation and Fate of Ion Pairs during MALDI Analysis: Anion Adduct Generation as an Indicative Tool to Determine Ionization Processes
}

\author{
Ralf Krüger and Michael Karas \\ Institute for Pharmaceutical Chemistry, Biocenter, J.-W. Goethe University Frankfurt, Frankfurt, Germany
}

\begin{abstract}
Stimulated by recent experiments, which verified the preservation of the analyte solution charge state upon incorporation in the host matrix crystals, investigations are reported focusing on the role of analyte and counter ions in the matrix-assisted laser desorption/ ionization (MALDI) process. These counter ions are only visible in the MALDI mass spectra under certain conditions, i.e., if inter-ionic proton transfer followed by evaporation of the neutrals is prevented, as in the case of metal cations. However, ion pairs can also survive the MALDI process if anions of very low gas phase basicities are used. By this means the intermediates of ion production in MALDI can be visualized. Depending on the amount of energy transfer to the analyte, which is mainly controlled by the matrix, different grades of adduct generation are observed. The analyte-, matrix- and polarity-dependant adduct distribution substantiates the hypothesis that multi-ion pairs are incorporated in the MALDI crystals and that ionization is essentially accomplished by charge separation processes. Moreover, the adduct distribution-and most probably also the charge separation efficiencywas found to be caused mainly by competition of different anionic species for coordination at the positively charged analyte sites. Furthermore, the results point to a less efficient charge separation with increasing number of ion pairs, which might be one major reason that mainly singly charged ions are obtained with MALDI. (J Am Soc Mass Spectrom 2002, 13, 1218-1226) (C) 2002 American Society for Mass Spectrometry
\end{abstract}

$\mathrm{T}$ The existence and preservation of charged compounds in the matrix crystals is a fundamental prerequisite of the hypothesis that the MALDI process is an ablative mechanism in which charged clusters are produced by charge separation processes throughout the rapid energy transfer to the sample [1]. It was shown recently that the solution charge state of the analyte is indeed maintained upon incorporation in MALDI matrices [2], thus counter ions have to be included in the crystals to account for charge balance. Consequently, cation and anion adducts should show up in MALDI mass spectra according to the number and polarity of charged groups of the analyte. However, whereas (metal) cation adducts are a common MALDI phenomenon, anion adducts are typically not observed. Anion-adduct formation is therefore the focus of this paper, because it promises to give insight into primary MALDI ionization mechanisms, e.g., formation, separation, and neutralization of ion pairs

Published online September 6, 2002

Address reprint requests to Dr. M. Karas, Institute for Pharmaceutical Chemistry, J.-W. Goethe University Frankfurt, Marie-Curie Str. 9, D-60439 Frankfurt, Germany. E-mail: karas@iachem.de during sample preparation and during the ablation process.

Analytes without basic or acidic groups such as oligosaccharides [3] and polymers [4] can only be detected either by cationization or anionization. Cationization efficiency can be enhanced by addition of metal salts [5-9] which is routinely used for detection of neutral compounds. One of the few examples of anionization is the detection of hydrogensulfate adducts of oligosaccharides by using sulfuric acid as additive, but also covalently bound sulfates (condensation reaction) are detected using higher laser fluences or higher sulfuric acid concentrations [10]. Adducts also occur with alkylsulfonates [11].

Ion-adduct formation is, however, not restricted to neutral species: even compounds with basic functions, which are mainly detected as $[\mathrm{M}+\mathrm{H}]^{+}$molecular ions in positive ion mode (e.g., peptides [12, 13] or nucleic acids [14, 15]), do form cation (e.g., alkaline-ion) adducts at least with minor intensities. Enhanced cation attachment is observed in particular if the analyte has negatively charged site(s) in solution, independent of the net charge, and many compounds are indeed zwitterionic. Especially sulfonate or phosphonate groups are deprotonated and thus negatively charged at least 
down to $\mathrm{pH}$ 1. Consequently, exchange of sodium against ammonium cations (which are able to neutralize the anions) is beneficial for nucleotides to avoid a broad distribution of cation adducts [16, 17]. Furthermore, doubly charged cations (e.g., $\mathrm{Cu}^{2+}[18-20]$ or $\mathrm{Zn}^{2+}$ [21, 22]) usually yield adducts of the form $[\mathrm{M}-\mathrm{H}+\mathrm{Cat}]^{+}$, suggesting the existence of ion pairs as well (although reduction to the monovalent cation adduct $[\mathrm{M}+\mathrm{Cat}]^{+}$ may occur, too).

Nevertheless, anion adducts are only rarely observed in MALDI. For example, Breuker et al. detected anion adducts of different biological compounds, but they used a special preparation method by mixing of silicon particles with tetrabutylammonium salts as ionization agents [23]. A better chance to detect adducts is given for mixtures of polycations and polyanions with higher (and nearly identical) masses, because more interionic attractions add up to a stronger interaction. For example, Juhasz and Biemann were able to detect complexes of highly acidic compounds bearing several negatively charged groups (e.g., suramin, heparines, or oligonucleotides) by adding multiply positively charged substances such as arginine-rich peptides or proteins as complexing agents [24]. In principle, the same complexation by salt bridges is valid in the case of noncovalent protein complexes, which can be detected with MALDI using appropriate nondenaturing conditions [25-29]. Another more recent example of anion adduct generation is the detection of protein-dye complexes with MALDI by Zenobi and coworkers [30-32]. The dyes used in their experiments contain sulfonic acid residues, which bind to positively charged sites in the protein, mainly guanidinium groups. Moreover, they were able to determine the number of arginines exposed to the protein surface by counting the number of anion adducts.

The detection of several adduct species in MALDI mass spectra is a strong hint for the existence of ion pairs in solution, their incorporation in the host matrix crystals, and their survival during the MALDI process, if neutralization by proton transfer is impossible. However, small anions attached to biomolecules are generally easily neutralized by proton transfer from neighbouring positive basic sites. Consequently, attachment of anions to positively charged analytes, e.g., proteins in acidic environment, is restricted to special conditions and is usually not observed with standard MALDI preparation protocols. It has to be mentioned that adducts of small anions with basic analytes are not necessarily unstable in the gas phase because these adducts are easily detected with ESI-MS [33, 34], where considerably less energy is transferred to the analyte molecules. With regard to the MALDI process, there are two possibilities to account for this differerence: First, it has to be considered that in the MALDI case more energy is transferred to the ion pairs, which can lead to enhanced proton transfer from the positive sites to the anions followed by evaporation of the generated neutral acids. Second, the exact position of the counter ions relative to the analyte molecules in the matrix crystal is unknown. Theoretically, the ion pairs can exist as closely bound contact ion pairs or as loosely bound solvent-separated ion pairs, where the distance between these ions can either be small (with just a few solvent molecules between them) or even quite far. These possibilities were already considered in a study concerning the existence of preformed ions in matrix crystals [35] and are also known to be influenced by solvation or hydrogen bonds, respectively [36]. Moreover, it has to be considered that matrix anions will compete for positive sites with anions added to or present in solution.

To find out the starting conditions of analyte ions and their counter ions in the matrix crystals, and to investigate the fate of these ion pairs upon ablation, different very strong acids were added to the analyte solution prior to sample preparation. The corresponding anions of these "superacids" have in common their gas phase basicities (GB) which are considerably lower compared to other common anions such as chloride or trifluoroacetate. Proton transfer from a protonated basic site to this anion is less exergonic and can even become (highly) endergonic because of coulombic forces which have to be overcome. Thus, proton transfer followed by subsequent evaporation of the neutral acid can be reduced or suppressed. The experiments were carried out with several different matrices in positive and negative ion mode for comparison. To avoid uncertainties related to the tertiary structure, all measurements were performed in strong acidic solutions $(\mathrm{pH}<2)$, so that protein denaturation was ensured and all basic (and acidic) sites were readily accessible.

\section{Experimental}

\section{Samples}

All acids and the standard matrices were obtained from Sigma-Aldrich (Deisenhofen, Germany), Fluka (Buchs, Switzerland), or Merck (Darmstadt, Germany). All substances were used as supplied commercially without further purification. The arginine containing peptides with the sequence AXG AXG AXG AXG (where $X$ is either arginine or threonine) were synthesized and HPLC-purified by WITA GmbH, Teltow, Germany. The matrix derivative $\alpha$-cyano-4-methoxycinnamic acid amide ( $\alpha$ CMCA) was synthesized in our laboratory by means of a piperidine catalyzed Knoevenagel condensation by slowly adding 4-methoxybenzaldehyde to cyanoacetamide at $0^{\circ} \mathrm{C}[37,38]$.

\section{Sample Preparation}

As standard solvent for analytes and matrices (except $\alpha$-cyano-4-hydroxycinnamic acid ( $\alpha \mathrm{CHCA}$ ) and its derivative $\alpha \mathrm{CMCA}$ ) a mixture of acetonitrile/water 1:1 (vol:vol) was used. The concentration of the matrix solutions was $0.1 \mathrm{M}$ and the concentration of the 
<smiles>[3H]C(F)(F)S(=O)(=O)NS(=O)(=O)C(F)(F)F</smiles>

Scheme 1. Structure of bis(trifluoromethylsulfonyl)imide (HTFSI).

analytes was $10^{-5}-10^{-4} \mathrm{M}$. The acids perchloric acid, hexafluorophosphoric acid, and bis(trifluoromethylsulfonyl)imide were dissolved in water at concentrations between 0.01 and $0.1 \mathrm{M}$. For MALDI standard dried droplet preparations, $1 \mu \mathrm{L}$ of each solution (matrix, analyte, and acid), were mixed on the MALDI target and dried by a gentle flow of air. The cinnamic acid matrices $\alpha \mathrm{CHCA}$ and $\alpha \mathrm{CMCA}$ were dissolved in acetone containing $5 \%$ water for surface preparations: A thin matrix film was produced, dried, and droplets of analyte and acid solution were mixed an the top of the matrix layer and dried to yield a uniform surface.

\section{Mass Spectrometry}

MALDI-MS experiments were carried out with a Voyager-DE PRO time-of-flight mass spectrometer (Applied Biosystems, Framingham, MA) with delayed extraction in the linear mode. The mass spectrometer is equipped with a nitrogen laser (Laser Science, Franklin, MA) emitting at $\lambda=337 \mathrm{~nm}$.

\section{Results and Discussion}

The experiments reported below were carried out in acidic solution at $\mathrm{pH}<2$ by using either perchloric acid $\left(\mathrm{HClO}_{4}\right)$, hexafluorophosphoric acid $\left(\mathrm{HPF}_{6}\right)$, or bis(trifluoromethylsulfonyl)imide (HTFSI [39, 40], structure depicted in Scheme 1). The corresponding anions of these strong acids have relatively low gas phase basicities (GB). Some known GB values of anions are compared in Table 1 (taken from http:/ / webbook.nist. gov/).

MALDI sample preparation with one of these acids lead to appearance of anion adducts in the mass spectra independant of which acid was used. Figure 1 shows MALDI spectra of ubiquitin using 2,5-dihydroxybenzoic acid (2,5-DHB) as matrix for each acid. In all spectra a similar number and distribution of anion adducts is visible, varying mass differences reflect the different anions. No adducts were found in control experiments upon addition of trifluoroacetic acid (TFA). If two anions with low gas phase basicities are present, e.g., TFSI and $\mathrm{ClO}_{4}^{-}$, mixed adducts are observed, too.

The multiple adduct formation is a strong hint that the multiply charged analyte cations are incorporated together with their counter ions in the matrix crystals. The untypical observation of strong anionization can be
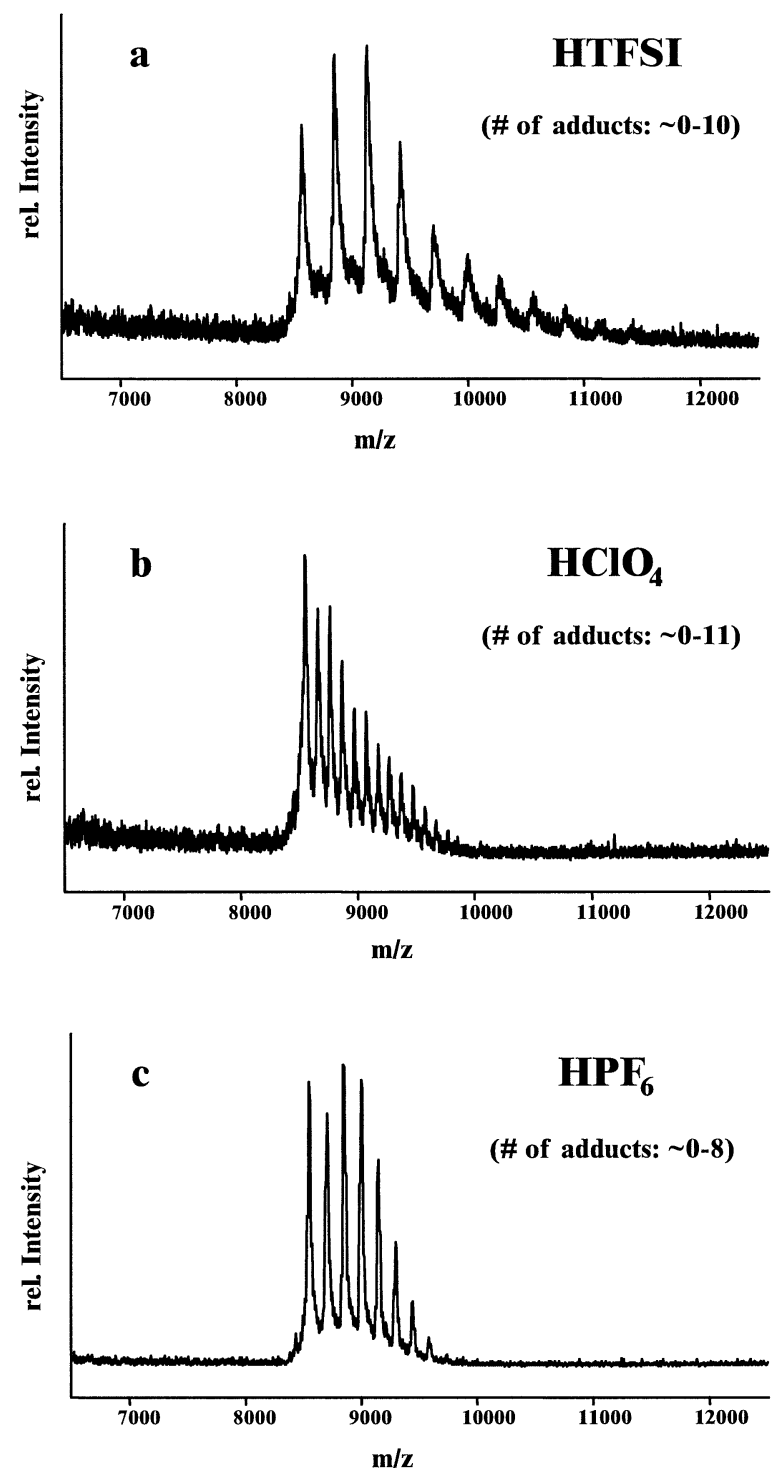

Figure 1. Mass spectra of ubiquitin prepared with 2,5-dihydroxybenzoic acid (2,5-DHB) and different strong acids: (a) Bis(trifluoromethylsulfonyl)imide (HTFSI); (b) $\mathrm{HClO}_{4} ;$ (c) $\mathrm{HPF}_{6}$.

explained by two different approaches: First, these anions might be more closely (and maybe more tightly) bound to the analyte than other anions. Second, charge neutralization by proton transfer is energetically unfavorable, especially in the case of anions with low gas phase basicities (GB). Although the first argument must not be neglected, the second requirement has to be met in any case and is therefore a necessary prerequisite for observation of anion adducts.

The influence of the GB can be described as follows: Anions have generally a higher GB than neutral bases due to the negative charge (some values are given in Table 1). Therefore, all neutralization reactions are exergonic, but coulombic forces have to be included, too. Ionic forces between formiate and protonated guanidine, imidazole, or ammonia as a model system have been calculated to be between 490 and $590 \mathrm{~kJ} / \mathrm{mol}$ at the 


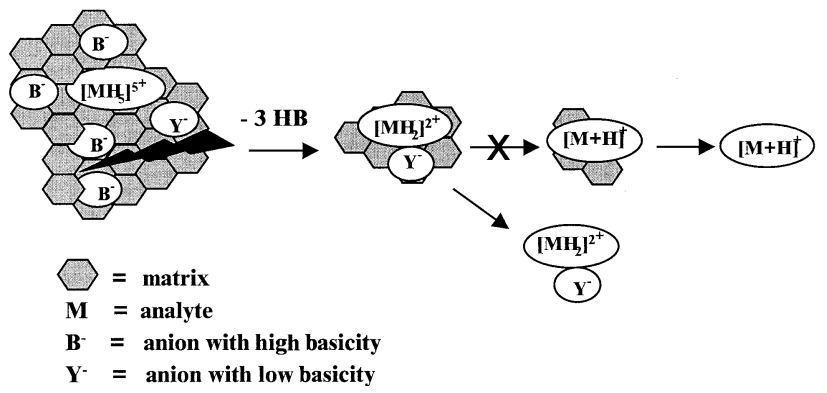

Scheme 2. Tentative mechanism of adduct formation with anions of low gas phase basicity.

minimum distance (250-390 pm) [36]. This interaction energy is reduced in a partially solvated system to $350-415 \mathrm{~kJ} / \mathrm{mol}$. This energy barrier has to be overcome by the energy release upon proton transfer for an ion pair to neutralize. If the proton transfer energy is considerably lowered, e.g., in the case of an ion pair between arginine and TFSI $(\Delta \mathrm{GB}=214 \mathrm{~kJ} / \mathrm{mol})$, the reaction will become highly endergonic because of electrostatic forces and the anion adduct survives the desorption process and becomes visible in the mass spectrum. The tentative mechanism of adduct generation is illustrated in Scheme 2: The analyte is incorporated in a multiply charged state together with the corresponding counter anions. Upon laser ablation, charged clusters are generated, either by charge separation and/or by photoionization processes, and neutralization and subsequent evaporation of neutrals lead to the final ion. However, in case of a weakly basic anion $\left(\mathrm{Y}^{-}\right)$inter-ionic proton transfer is prohibited and the adduct will survive the MALDI process.

\section{Influence of Matrix and Analyte}

The maximum number, the distribution, and the peak intensities of anion adducts depend not only on the analyte, but also on the matrix. With most matrices, e.g., 2,5-DHB or $\alpha \mathrm{CHCA}$, only a few adducts were observed, presumably mainly arginine residues are complexed; this was found before by Zenobi and coworkers for a similar system [30-32]. Anionization is, nevertheless, not restricted to arginine residues because with increasing mass the number of adducts exceeds the number of arginines as can be seen in Figure 2. Cytochrome $C$ has only two arginines in its sequence, but many more adducts are detected with 2,5-DHB, although the first peak is still remarkably higher than the others.

However, if the matrix 3-hydroxypicolinic acid (3HPA) is used instead, a strong shift to higher adduct numbers is observed, and the maximum adduct number in the positive-ion mode corresponds to the number of all basic sites in the molecule minus 1 (see spectra of bovine insulin in Figure 3a and b). Besides one arginine, one lysine, and two N-termini two histidines are also protonated in a strongly acidic environment. The correlation of the adduct number with the number of basic sites was consistently observed for a variety of peptides

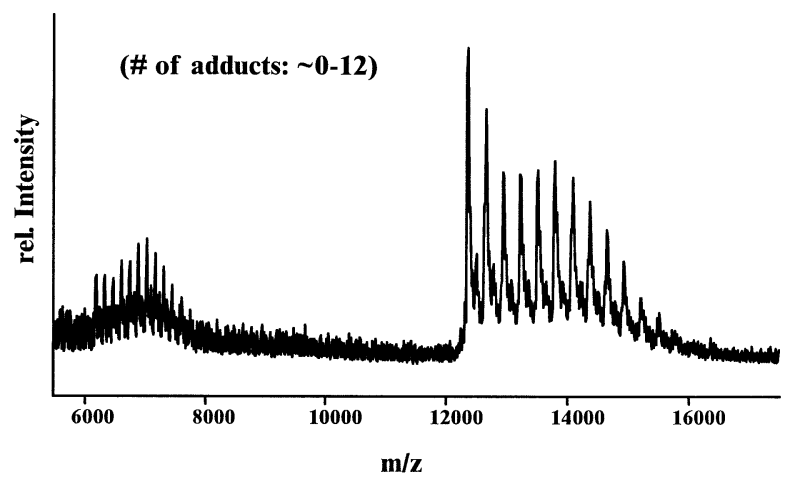

Figure 2. Mass spectrum of cytochrome $C$ prepared with 2,5dihydroxybenzoic acid (2,5-DHB) and bis(trifluoromethylsulfonyl)imide (HTFSI).

and small proteins. For cytochrome C only an unresolved broad signal of minute intensity was observed with 3-HPA, corresponding to the high number and broad distribution of anion adducts resulting from the 24 basic sites and presumably also due to their metastable fragmentation.

The method can be used to estimate the total number of basic sites as well as the number of arginine residues. The spectra in Figure 4 obtained with two different matrices show a mixture of three peptides with different basic sites: Bradykinin (2 Arg and $1 \mathrm{~N}$-terminus), neurotensin (2 Arg and 1 Lys), and [Glu $\left.{ }^{1}\right]$-fibrinopeptide (1 Arg and $1 \mathrm{~N}$-terminus). In the first spectrum, using 2,5-DHB as matrix, one adduct of bradykinin $(\mathrm{m} / \mathrm{z}$ $1060)$ and one adduct of neurotensin $(\mathrm{m} / \mathrm{z}$ 1673) can be detected by the characteristic mass shift of $m / z 281$, but no adduct of $\left[\mathrm{Glu}^{1}\right]$-fibrinopeptide $(\mathrm{m} / \mathrm{z} 1570)$ is visible. The observation agrees well with the fact that $\left[\mathrm{Glu}^{1}\right]$ fibrinopeptide has only one arginine residue whereas the other peptides have two. The spectrum recorded with 3-HPA shows two adducts of bradykinin and two adducts of neurotensin, in accordance with the existence of three basic residues in each peptide, but only one adduct of $\left[\mathrm{Glu}^{1}\right]$-fibrinopeptide with only two basic residues (1 Arg, $1 \mathrm{~N}$-terminus) appears.

It is noticeable that in all the spectra recorded with 3-HPA the signal corresponding to the highest number of anion adducts is only detected with remarkably lower intensity. One can speculate that this last anion is coordinated to a site of low basicity, i.e., the N-terminus, and that it is more efficiently neutralized within the desorption process. This would suggest that the excess charge is not located at the $\mathrm{N}$-terminus and that starting from a neutral multi-ion pair, i.e., from an ion cluster with a net charge of zero, charge separation occurs preferably at basic sites other than the N-terminus, maybe because of different ion pair distances or energetic demands.

The observations can be used to estimate the point where complexes are no longer observed (GB values are given in Table 1). For most matrices used except 3-HPA, this value should be higher than $214 \mathrm{~kJ} / \mathrm{mol}$, which is 

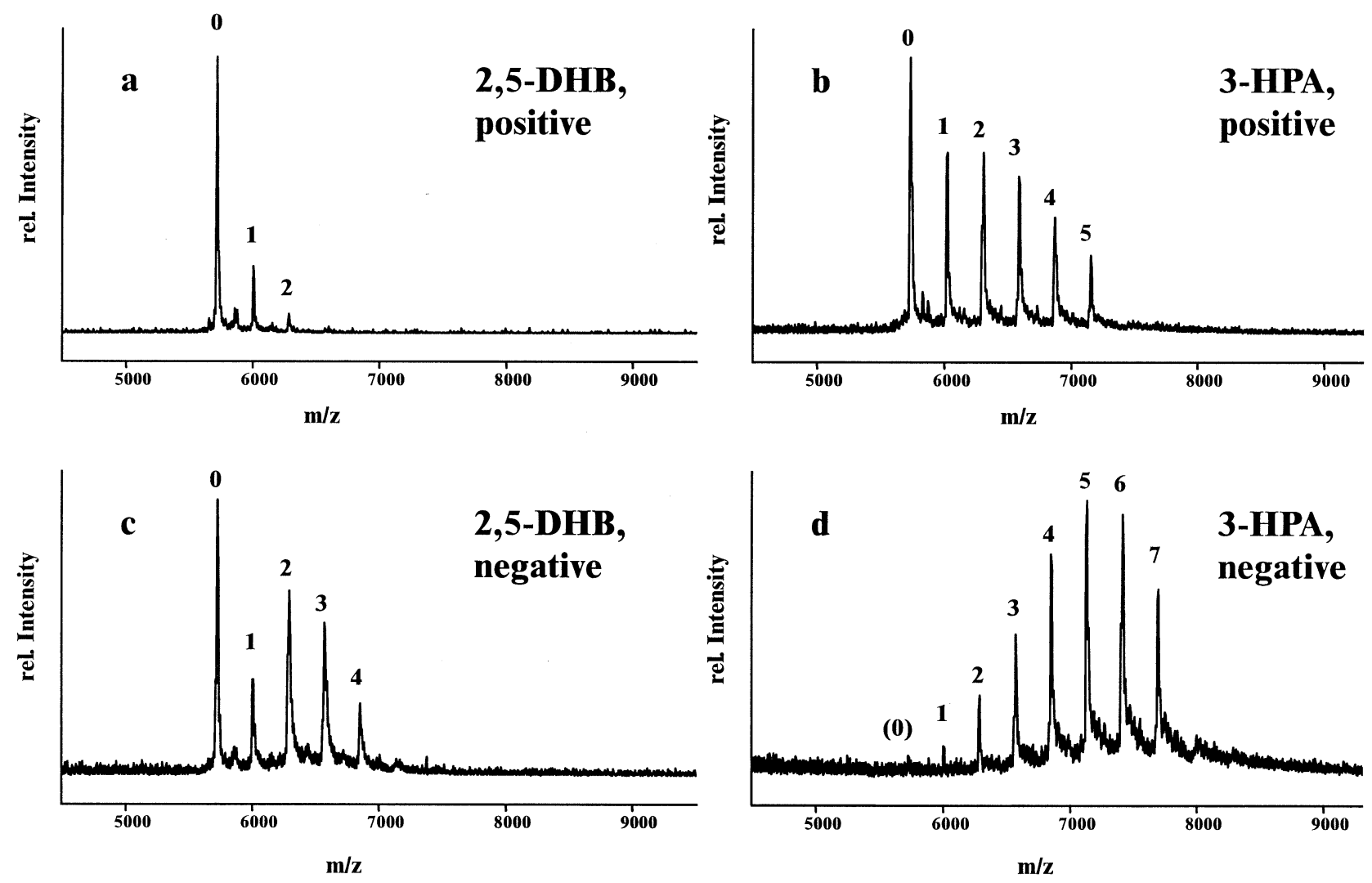

Figure 3. Mass spectra of bovine insulin in positive and negative ion mode obtained with two different matrices and addition of HTFSI \{bis(trifluoromethylsulfonyl)imide\} (a) 2,5-DHB (2,5dihydroxybenzoic acid), positive; (b) 3-HPA (3-hydroxypicolinic acid), positive; (c) 2,5-DHB, negative;

(d) 3-HPA, negative. The peak labels refer to the number of TFSI adducts.

the $\Delta \mathrm{GB}$ of the ion pair arginine/TFSI, because these are always detected. As partial complexation of histidine and lysine residues occurs at least for analytes with higher masses, the barrier should be in the order of $250-350 \mathrm{~kJ} / \mathrm{mol}(\Delta \mathrm{GB}$ Lysine $/$ TFSI $=269 \mathrm{~kJ} / \mathrm{mol})$. Assuming an average $\Delta \mathrm{GB}$ limit of $300 \mathrm{~kJ} / \mathrm{mol}$ above which adducts cannot be observed, subtraction of the coulombic term (see previous chapter and references [36]) delivers an estimate of the activation energy necessary for adduct decomposition. This amounts to 190$290 \mathrm{~kJ} / \mathrm{mol}$ neglecting solvation effects, but drops to $50-115 \mathrm{~kJ} / \mathrm{mol}$ in case of solvated ion pairs, however, a considerable uncertainty remains.

In contrast, the "softer" matrix 3-HPA leads to enhanced adduct survival so that slightly higher $\Delta \mathrm{GB}$ differences are tolerated without adduct decompositon, which points to a lower degree of activation upon the desorption process. The softness of the matrix 3-HPA becomes visible by a low degree of fragmentation and can be correlated to a higher initial ion velocity compared to other matrices [1]. The reason might be the more effective expansional cooling upon the ablation process. However, no adducts are observed between arginine and TFA, although the GB difference is only $318 \mathrm{~kJ} / \mathrm{mol}$. This discrepancy is a hint that not only GB differences are responsible for all of the observed effects and that additional energetic terms such as hydrogen bridges or solvation effects have to be considered. Coulomb attractions are significantly lowered upon solvation of ion pairs [36], and closer contact or a reduced solvation might lead to enhanced adduct formation using 3-HPA.

\section{Influence of Ion Polarity and Analyte Net Charge}

The spectra presented so far were all recorded in positive-ion mode. In a surprisingly general consistency for all cases investigated, the maximum number of detected anion adducts and the distributions are shifted by " +2 " in the negative ion mode. Again, this can be used to confirm data about the number of basic residues, because the difference of two adducts between positive and negative mode can be easily checked. It is noteworthy that this difference of two adducts corresponds to the same maximum number of protonated sites. The overall charge is caused by the different number of adducts only, with one anion deficit in positive ion mode and one anion excess in negative ion mode, respectively. For example, Figure 3 shows insulin spectra recorded in negative and positive ion mode: Up to seven adducts are detected with 3-HPA in negative ion mode (see Figure 3d); since insulin has only six basic residues, this is a clear case of anionization for which the "ionizing" excess anion needs to be bound unspe- 
Table 1. Gas phase basicities (BG) of some anions and neutral bases

\begin{tabular}{lc}
\hline & $\mathrm{GB}(\mathrm{kJ} / \mathrm{mol})^{\mathrm{a}}$ \\
\hline \hline Anion & \\
$\mathrm{CH}_{3} \mathrm{COO}^{-}$ & 1429 \\
$\mathrm{Glycine}^{-}$ & 1404 \\
$\mathrm{Cl}^{-}$ & 1373 \\
$2,5-\mathrm{DHB}^{-}$ & 1329 \\
$\mathrm{CF}_{3} \mathrm{COO}^{-}$ & 1325 \\
$\mathrm{HSO}_{4}^{-}$ & 1265 \\
$\mathrm{TFSI}^{-}$ & 1221 \\
$\mathrm{ClO}_{4}^{-}$ & 1180 \\
$\mathrm{Neutral}^{-}$base & \\
Arginine (side chain) & 1007 \\
Lysine (side chain) & 952 \\
$\mathrm{Histidine}$ (side chain) & 952 \\
$\mathrm{Glycine}^{-N}$-terminus) & 852 \\
$\mathrm{NH}_{3}$ & 847 \\
$\mathrm{H}_{2} \mathrm{O}$ & 685 \\
\hline
\end{tabular}

${ }^{a}$ Values are taken from http://webbook.nist.gov/

cifically to the multi-ion pair. Any addition of a second anion to form doubly-charged ions will be improbable because of the coulombic repulsion and the low interaction energy, conversely the separation of a second anion to form doubly-charged positive ions is made more difficult by coulomb attraction. In this simple way, the production of mainly singly charged ions by
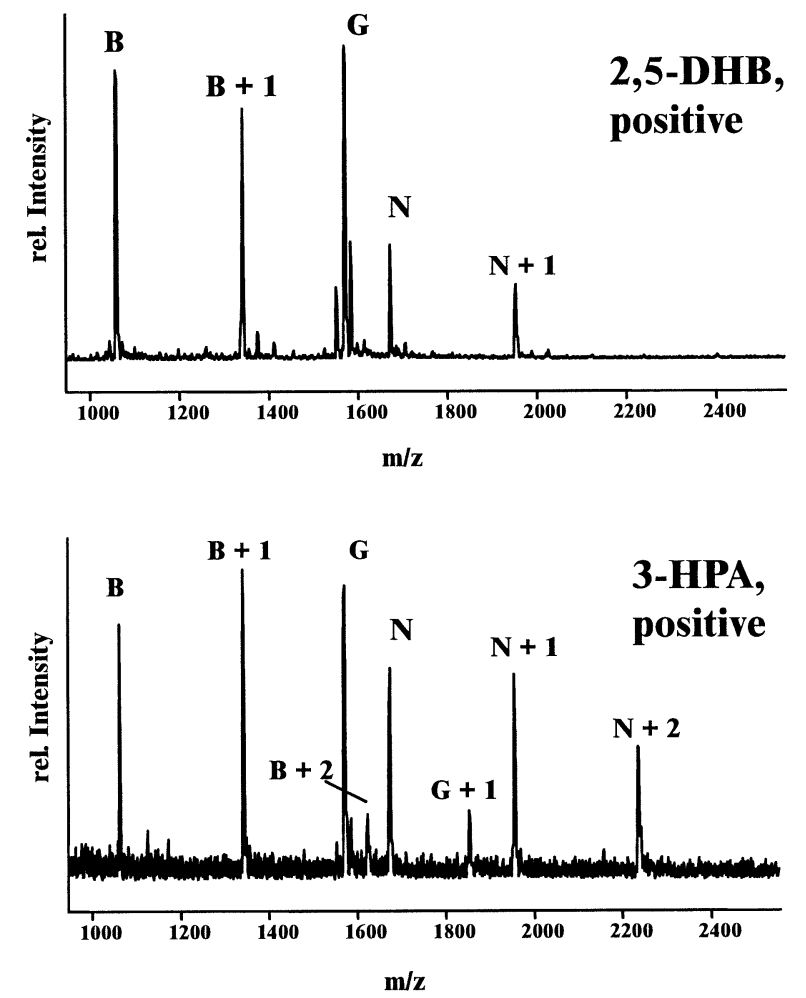

Figure 4. Positive mass spectra of a peptide mixture obtained with two different matrices and addition of HTFSI \{bis(trifluoromethylsulfonyl)imide $\}$. B = bradykinin, $\mathrm{G}=\left[\mathrm{Glu}^{1}\right]$-fibrinopeptide, $\mathrm{N}=$ neurotensin; the numbers refer to the number of TFSI-adducts.
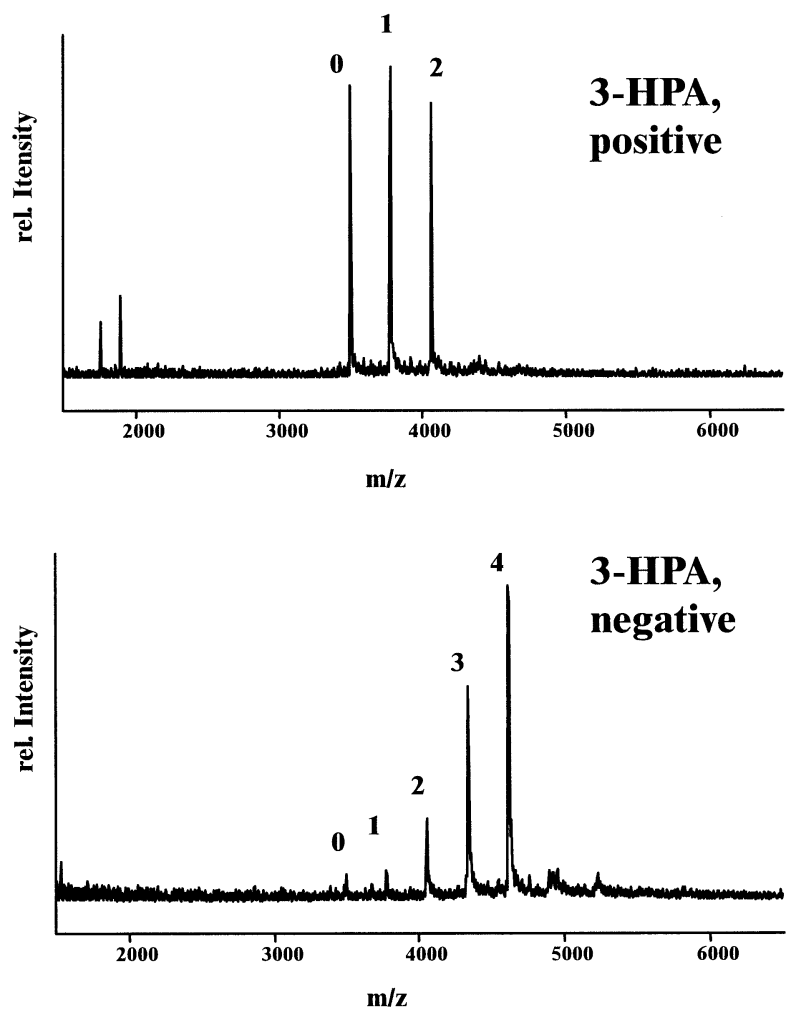

Figure 5. Mass spectra of oxidized bovine insulin B-chain obtained with 3-HPA (3-hydroxypicolinic acid) and HTFSI \{bis(trifluoromethylsulfonyl)imide\} in positive and negative ion mode. The peak labels refer to the number of TFSI adducts.

MALDI can be rationalized. The majority of the clusters and thus analytes might indeed be completely neutralized by intact ion pairs and will not show up in the mass spectrum, and correspondingly an estimate to which extent charged species are formed is not possible.

In a further experiment, the influence of the net charge state of the analyte was investigated. All samples were prepared in acidic environment so that carboxylic acids were protonated and thus not negatively charged. However, phosphoric or sulfonic acid residues will still be negatively charged. Figure 5 depicts the mass spectrum of oxidized bovine insulin B-chain using 3-HPA as a matrix. Less adducts than expected from the number of basic sites alone are visible: Insulin B-Chain has five basic residues (1 Arg, 1 Lys, 2 His, and 1 N-terminus), but also contains two sulfonates because of the oxidized cysteines. The fact that the maximum number of anion adducts is reduced by two in both polarities clearly indicates that intermolecular salt bridges between basic sites and sulfonates exist, resulting in a reduced net charge of the analyte and consequently less adduct formation.

\section{Anion Competition and Charge Separation Efficiency}

All analytes show a distribution of anion adducts, if weakly basic anions are added, up to the maximum 

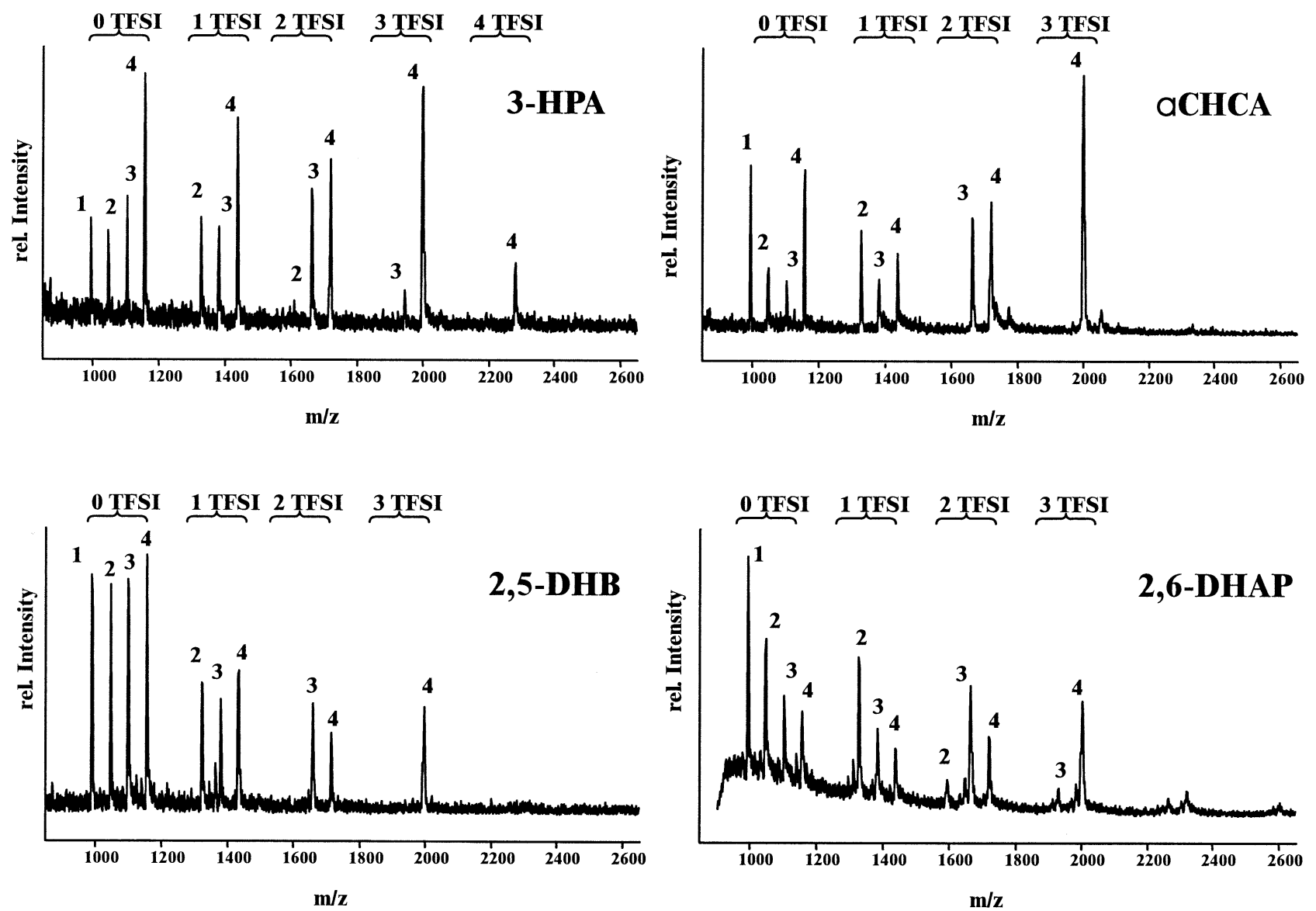

Figure 6. Positive mass spectra of a peptide mixture obtained with different matrices and addition of HTFSI \{bis(trifluoromethylsulfonyl)imide\}. The peptides have the sequence AXG AXG AXG AXG with $X=$ Arg or Thr, and the peak labels refer to the number of arginines in the peptide. The peaks are grouped according to the number of TFSI adducts. (3-HPA $=3$-hydroxypicolinic acid; $\alpha \mathrm{CHCA}=$ $\alpha$-cyano-4-hydroxycinnamic acid; 2,5-DHB = 2,5-dihydroxybenzoic acid; 2,6-DHAP = 2,6-dihydroxyacetophenone).

possible number. That typically a distribution of anion adducts is observed and not just the maximum-number species-which would to be expected from a simple charge-separation model-can be attributed to two effects. First, analyte ions are excited due to the MALDI process, which supplies the energy to overcome the neutralization barrier also in case of endergonicity. Since the degree of excitation depends on the matrix, different degrees of adduction are observed, with a shift to a higher number of anion adducts for softer matrices, such as 3-HPA [1]. Second, at $\mathrm{pH}$ values below the $\mathrm{pKa}$ of the matrix, a percentage of the excess matrix still exists in its anionic form; these anions compete with the weakly basic test anions for coordination to a protonated positive site. Since their neutralization is by far less endergonic or even exergonic, these ion pairs will not show up.

These assumptions were tested with matrices of different acidities. Figure 6 shows spectra of an equimolar peptide mixture doped with HTFSI. The peptides have the general formula AXG AXG AXG AXG, where $X$ is either threonine or arginine; they differ by the number of arginines and thus positive charges. Using 2,5-DHB, $\alpha \mathrm{CHCA}$, or 2,6-dihydroxyacetophenone (2,6DHAP), the number of anion adducts visible in the spectra correlates well with the number of arginines in the peptide, while with 3-HPA some N-terminal anion adducts also appear with low abundance, as pointed out above. All acidic matrices lead to a broad distribution of anion adducts and quantitative differences are visible, however, these are not easily explainable. For example, the prominent shift to lower-number adducts in the case of 2,5-DHB may also be induced by the crystallization process and a more pronounced exclusion of the test anion compared to other matrices.

As the extreme case, a matrix without basic or acidic functional groups is investigated. A derivative of $\alpha \mathrm{CHCA}, \quad \alpha$-cyano-4-methoxycinnamic acid amide $(\alpha C M C A)$, was chosen; now TFSI is the only anion in the solution. Indeed, both in positive- and negative-ion mode the maximum-number anion adducts are observed nearly exclusively, however, with reasonable ion intensities in the positive-ion mode for all peptides (see Figure 7a). In the negative-ion mode only the most basic 

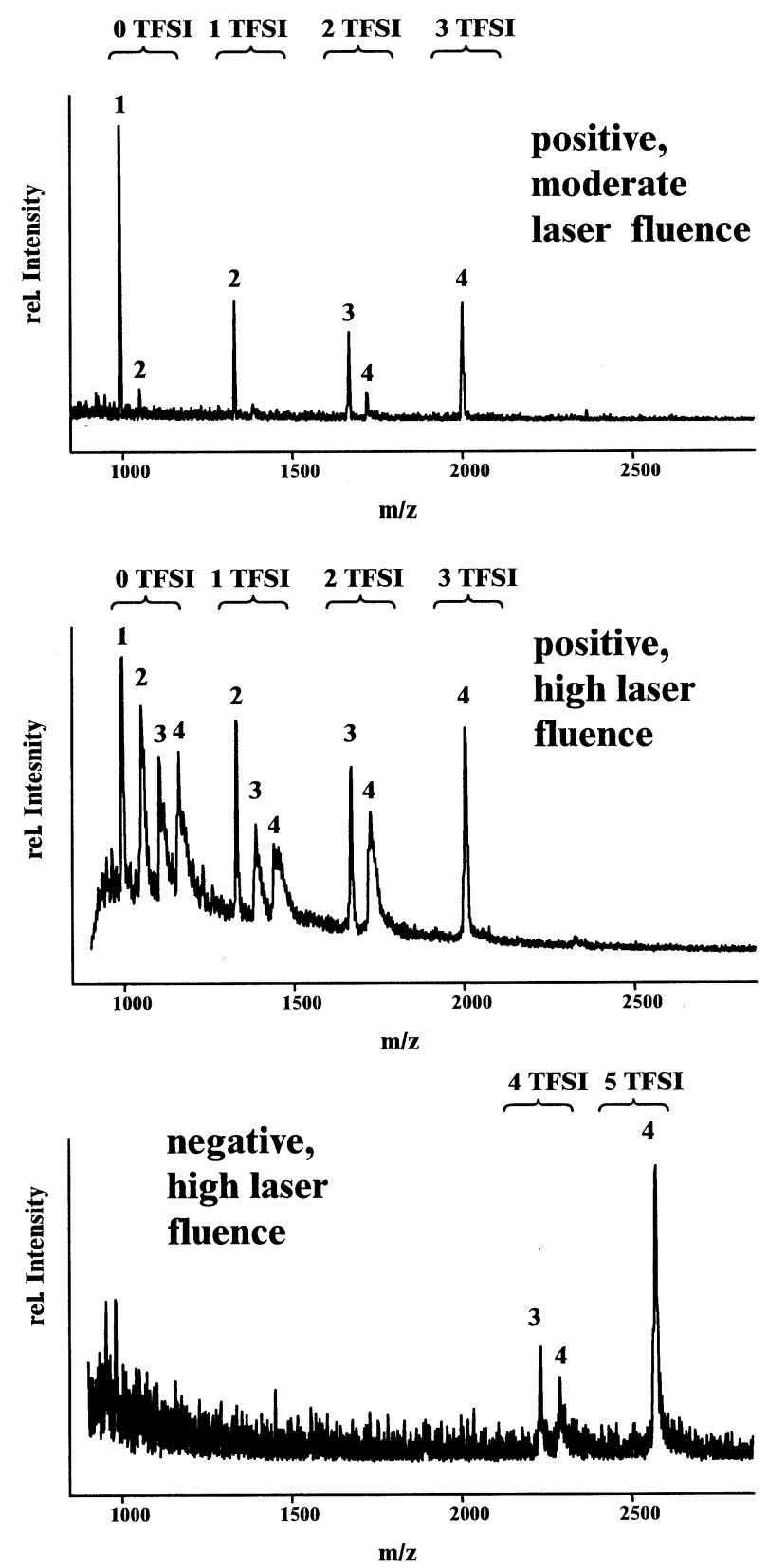

Figure 7. Mass spectra of a peptide mixture obtained with $\alpha$-cyano-4-methoxycinnamic acid amide ( $\alpha \mathrm{CMCA})$ and addition of bis(trifluoromethylsulfonyl)-imide (HTFSI). The peptide sequences and the peak labels are explained in Figure 6.

peptides are detected with low signal-to-noise ratio (Figure 7c). This points to the role of the number of basic sites in binding the excess anion, even if those are compensated formally by counter ions. Conversely, the stronger attractions with every additional basic site also lead to a less efficient charge separation, visible by the constant or even decreasing signal intensities observed in the positive ion mode. It is noteworthy that the opposite intensity distribution is observed if acidic matrices are used, especially if all signal intensities related to the same peptide are added up (see Figure 6). This effect can be rationalized assuming that the more demanding charge separation for more highly charged (basic) analytes in case of stable ion pairs would be overcompensated by the easier charge separation in the case of matrix counter anions and an enhanced probability for a higher number of basic sites. But not only anion competition is responsible for the signal distribution, because also at high laser fluences a shift to smaller adduct numbers is induced due to (metastable) decay (Figure $7 \mathrm{~b}$ ). These observations support the important role of both analyte-ion excitation and (matrix) anion competition for the generation of adduct distributions as discussed above.

\section{Conclusions}

The detection of anion adduct distributions corresponding to the number of basic sites in the analyte by using weakly acidic anions supports the assumption that analytes are incorporated as (multiply) charged ions with their counter ions in the host matrix crystal, and substantiates the idea that MALDI ions are generated by an explosive cluster charge separation mechanism. There are two effects which influence the possible product ion distribution: First, the matrix-dependant amount of energy transfer to the analyte and second, the competition of different counter ions for the charged analyte sites. Moreover, the efficiency of charge separation and thus ion production is determined by the way of ion pairing, which is certainly not only influenced by the existing ions themselves but also by the crystallization procedure. Therefore, each strategy to improve MALDI analysis has to deal with-and has been dealing with-empirical optimization of sample preparation procedures, e.g., the choice of solvents or additives.

\section{References}

1. Karas, M.; Glückmann, M.; Schäfer, J. Ionization in MatrixAssisted Laser Desorption/Ionization: Singly Charged Molecular Ions are the Lucky Survivors. J. Mass Spectrom. 2000, 35, $1-12$.

2. Krüger, R.; Pfenninger, A.; Fournier, I.; Glückmann, M.; Karas, M. Analyte Incorporation and Ionization in Matrix-Assisted Laser Desorption/Ionization Visualized by $\mathrm{pH}$ Indicator Molecular Probes. Anal. Chem. 2001, 73, 5812-5821.

3. Harvey, D. J. Matrix-Assisted Laser Desorption/Ionization Mass Spectrometry of Carbohydrates. Mass Spectrom. Rev. 1999, 18, 349-451.

4. Nielen, M. W. F. MALDI Time-of-Flight Mass Spectrometry of Synthetic Polymers. Mass Spectrom. Rev. 1999, 18, 309-344.

5. Bahr, U.; Deppe, A.; Karas, M.; Hillenamp, F. Mass Spectrometry of Synthetic Polymers by UV-Matrix-Assisted Laser Desorption/Ionization. Anal. Chem. 1992, 64, 2866-2869.

6. Dogruel, D.; Nelson, R. W.; Williams, P. The Effect of Matrix $\mathrm{pH}$ and Cation Availability on the Matrix-Assisted Laser Desorption/Ionization Mass Spectrometry of Poly(methylmethacrylate). Rapid Commun. Mass Spectrom. 1996, 10, 801804.

7. Pastor, S. J.; Wilkins, C. L. Analysis of Hydrocarbon Polymers by Matrix-Assisted Laser Desorption/Ionization-Fourier Transform Mass Spectrometry. J. Am. Soc. Mass Spectrom. 1997, $8,225-233$. 
8. Mowat, I. A.; Donovan, R. J.; Maier, R. R. J. Enhanced Cationization of Polymers Using Delayed Ion Extraction with Matrix-Assisted Laser Desorption/Ionization. Rapid Commun. Mass Spectrom. 1997, 11, 89-98.

9. Pfenninger, A.; Karas, M.; Finke, B.; Stahl, B.; Sawatzki, G. Matrix Optimization for Matrix-Assisted Laser Desorption/ Ionization Mass Spectrometry of Oligosaccharides from $\mathrm{Hu}-$ man Milk. J. Mass Spectrom. 1999, 34, 98-104.

10. Wong, A. W.; Cancilla, M. T.; Voss, L. R.; Lebrilla, C. B. Anion Dopant for Oligosaccharides in Matrix-Assisted Laser Desorption/Ionization Mass Spectrometry. Anal. Chem. 1999, 71, 205-211.

11. Wong, A. W.; Wang, H.; Lebrilla, C. B. Selection of Anionic Dopant for Quantifying Desialylation Reactions with MALDIFTMS. Anal. Chem. 2000, 72, 1419-1425.

12. Wang, B. H.; Dreisewerd, K.; Bahr, U.; Karas, M.; Hillenkamp, F. Gas-Phase Cationization and Protonation of Neutrals Generated by Matrix-Assisted Laser Desorption. J. Am. Soc. Mass Spectrom. 1993, 4, 393-398.

13. Liao, P.-C.; Allison, J. Ionization Processes in Matrix-Assisted Laser Desorption/Ionization Mass Spectrometry: Matrix-Dependent Formation of $[\mathrm{M}+\mathrm{H}]^{+}$versus $[\mathrm{M}+\mathrm{Na}]^{+}$Ions of Small Peptides and Some Mechanistical Comments. J. Mass Spectrom. 1995, 30, 408-423.

14. Shaler, T. A.; Wickham, J. N.; Sannes, K. A.; Wu, K. J.; Becker, C. H. Effect of Impurities on the Matrix-Assisted Laser Desorption Mass Spectra of Single-Stranded Oligodeoxynucleotides. Anal. Chem. 1996, 68, 576-579.

15. Nordhoff, E.; Kirpekar, F.; Roepstorff, P. Mass Spectrometry of Nucleic Acids. Mass Spectrom. Rev. 1996, 15, 67-138.

16. Cheng, S.-W.; Chan, T.-W. Use of Ammonium Halides as Comatrices for Matrix-Assisted Laser Desorption/Ionization Studies of Oligonucleotides. Rapid Commun. Mass Spectrom. 1996, 10, 907-910.

17. Zhu, Y. F.; Taranenko, N. I.; Allman, S. A.; Haff, L.; Chen, C. H. The Effect of Ammonium Salt and Matrix in the Detection of DNA by Matrix-Assisted Laser Desorption/Ionization Timeof-Flight Mass Spectrometry. Rapid Commun. Mass Spectrom. 1996, 10, 1591-1596.

18. Nelson, R. W.; Hutchens, T. W. Mass Spectrometric Analysis of a Transition-Metal-Binding Peptide Using Matrix-Assisted Laser Desorption/Ionization Time-of-Flight Mass Spectrometry. A Demonstration of Probe Tip Chemistry. Rapid Commun. Mass Spectrom. 1992, 6, 4-8.

19. Wong, C. K. L.; Chan, T.-W. D. Cationization Processes in Matrix-Assisted Laser Desorption/Ionization Mass Spectrometry: Attachment of Divalent and Trivalent Metal Ions. Rapid Commun. Mass Spectrom. 1997, 11, 513-519.

20. Salih, B.; Masselon, C.; Zenobi, R. Matrix-Assisted Laser Desorption/Ionization Mass Spectrometry of Noncovalent Protein-Transition Metal Ion Complexes. J. Mass Spectrom. 1998, 33, 994-1002.

21. Woods, A. S.; Buchsbaum, J. C.; Worrall, T. A.; Berg, J. M.; Cotter, R. J. Matrix-Assisted Laser Desorption/Ionization of Noncovalently Bound Compounds. Anal. Chem. 1996, 68, 576-579.

22. Lehmann, E.; Zenobi, R.; Vetter, S. Matrix-Assisted Laser Desorption/Ionization Mass Spectra Reflect Solution-Phase Zinc Finger Peptide Complexation. J. Am. Soc. Mass Spectrom. 1993, 4, 393-398.

23. Breuker, K.; Knochenmuss, R.; Zenobi, R. Matrix-Assisted Laser Desorption/Chemical Ionization with Reagent Ion Generation Directly from a Liquid Matrix. Int. J. Mass Spectrom. 1998, 176, 149-159.
24. Juhasz, P.; Biemann, K. Mass Spectrometric Molecular-Weight Determination of Highly Acidic Compounds of Biological Significance via their Complexes with Basic Polypeptides. Proc. Natl. Acad. Sci. U.S.A. Biochemistry 1994, 91, 4333-4337.

25. Cohen, L. R. H.; Strupat, K.; Hillenkamp, F. Analysis of Quaternary Protein Ensembles by Matrix-Assisted Laser Desorption/Ionization Mass Spectrometry. J. Am. Soc. Mass Spectrom. 1997, 8, 1046-1052.

26. Moniatte, M.; Lesieur, C.; Vécsey-Semjén, B.; Buckley, J. T.; Pattus, F.; van der Goot, F. G.; Van Dorsselaer, A. MatrixAssisted Laser Desorption/Ionization Time-of-Flight Mass Spectrometry in the Subunit Stoichometry Study of HighMass Noncovalent Complexes. Int. J. Mass Spectrom. Ion Processes 1997, 169/170, 179-199.

27. Farmer, T. B.; Caprioli, R. M. Determination of Protein-Protein Interactions by Matrix-Assisted Laser Desorption/Ionization Mass Spectrometry. J. Mass Spectrom. 1998, 33, 697-704.

28. Jespersen, S.; Niessen, W. M. A.; Tjaden, U. R.; van der Greef, J. Basic Matrices in the Analysis of Noncovalent Complexes by Matrix-Assisted Laser Desorption/Ionization Mass Spectrometry. J. Mass Spectrom. 1998, 33, 1088-1093.

29. Vogl, T.; Roth, J.; Sorg, C.; Hillenkamp, F.; Strupat, K. Calcium-Induced Noncovalently Linked Tetramers of MRP8 and MRP14 Detected by Ultraviolet Matrix-Assisted Laser Desorption/Ionization Mass Spectrometry. J. Am. Soc. Mass Spectrom. 1999, 10, 1124-1130.

30. Salih, B.; Zenobi, R. MALDI Mass Spectrometry of DyePeptide and Dye-Protein Complexes. Anal. Chem. 1998, 70, 1536-1543.

31. Friess, S. D.; Zenobi, R. Protein Structure Information from Mass Spectrometry? Selective Titration of Arginine Residues by Sulfonates. J. Am. Soc. Mass Spectrom. 2001, 12, 810-818.

32. Friess, S. D.; Daniel, J. M.; Hartmann, R.; Zenobi, R. Mass Spectrometric Noncovalent Probing of Amino Acids in Peptides and Proteins. Int. J. Mass Spectrom., 2002, 219, 269-281.

33. Mirza, U. A.; Chait, B. T. Effects of Anions on the Positive Ion Electrospray Ionization Mass Spectra of Peptides and Proteins. Anal. Chem. 1994, 66, 2898-2904.

34. Wang, G.; Cole, R. B. Effects of Solvent and Counterion on Ion Pairing and Observed Charge States of Diquaternary Ammonium Salts in Electrospray Ionization Mass Spectrometry. J. Am. Soc. Mass Spectrom. 1996, 7, 1050-1058.

35. Lehmann, E.; Knochenmuss, R.; Zenobi, R. Ionization Mechanisms in Matrix-Assisted Laser Desorption/Ionization Mass Spectrometry: Contribution of Preformed Ions. Rapid Commun. Mass Spectrom. 1997, 11, 1483-1492.

36. Fülscher, M.; Mehler, E. L. The Effect of Hydrogen Bonding on Protonation Energies and Ion Pair Formation. Chem. Phys. 1996, 204, 403-410.

37. Berlin, K.; Gut, I. G. Analysis of Negatively "Charge Tagged" DNA by Matrix-Assisted Laser Desorption/Ionization Timeof-Flight Mass Spectrometry. Rapid Commun. Mass Spectrom. 1999, 13, 1739-1743.

38. Schäfer, J. Ph.D. Thesis, J.-W. Goethe University, Frankfurt, 2000, 42.

39. Foropoulos, J., Jr.; DesMarteau, D. D. Synthesis, Properties, and Reactions of Bis\{(trifluoromethyl)sulfonyl $\}$ Imide, $\left(\mathrm{CF}_{3} \mathrm{SO}_{2}\right)_{2} \mathrm{NH}$. Inorg. Chem. 1984, 23, 3720-3723.

40. Rey, I.; Johansson, P.; Lindgren, J.; Lassègues, J. C.; Grondin, J.; Servant, L. Spectroscopic and Theoretical Study of $\left(\mathrm{CF}_{3} \mathrm{SO}_{2}\right)_{2} \mathrm{~N}^{-}\left(\mathrm{TFSI}^{-}\right)$and $\left(\mathrm{CF}_{3} \mathrm{SO}_{2}\right)_{2} \mathrm{NH}$ (HTFSI). J. Phys. Chem. A. 1998, 102, 3249-3258. 